\title{
The human proteome on target
}

SRMAtlas contains mass spectrometry assays allowing targeted analysis of nearly $100 \%$ of human proteins.

'Shotgun' mass-spectrometry-based proteomics approaches are indispensable for high-throughput protein discovery. However, because of an inherent instrumental bias toward detection of high-abundance peptides, shotgun analysis suffers from somewhat limited quantitative reproducibility.

In contrast, the targeted proteomics technique of selected reaction monitoring, or SRM, enables highly sensitive, accurate, quantitative analysis of selected proteins across multiple samples. In this technique, the mass spectrometer is programmed with 'SRM assays' to detect specific proteotypic peptides that are unique to the targeted proteins. This approach is akin to the lab workhorse method of western blotting, but it is more readily multiplexed and provides better specificity, quantitative ability, and reproduc- ibility than antibody-based methods. And an SRM assay can be used again and again, in any type of study, for any type of sample.

In contrast to shotgun methods, SRM data analysis is relatively straightforward. A bottleneck that has prevented its broader adoption, however, has been the lack of availability of SRM assays, which must be developed through a very tedious process that requires generating empirical mass spectrometry data for individual, synthetic proteotypic peptides.

Robert Moritz of the Institute for Systems Biology in Seattle, Ruedi Aebersold of ETH Zurich, and their colleagues have been working for many years to streamline this development process. They now present a proteomescale human resource that contains 158,015 SRM assays, which together target $99.7 \%$ of human proteins - that is, all but 22 of the UniProtKB/Swiss-Prot-annotated 20,277 human proteins. The large majority of proteins are covered by at least five SRM assays targeting different proteotypic peptides. The database also includes assays for more than 14,000 protein isoforms in total.

Targeted proteomics analysis allows researchers to focus in on specific groups of proteins, such as those in a particular signaling pathway. The Moritz-Aebersold team deployed SRM assays to monitor the effects of drug treatment in liver cell lines, where they studied the cholesterol biosynthesis pathway, and in three differentially responsive prostate cancer cell lines.

The human SRM assay resource joins assays for Saccharomyces cerevisiae and Mycobacterium tuberculosis, all made freely available via SRMAtlas at http://www. srmatlas.org.

\section{Allison Doerr}

\section{RESEARCH PAPERS}

Kusebauch, U. et al. Human SRMAtlas: a resource of targeted assays to quantify the complete human proteome. Cell 166, 766-778 (2016). 